\title{
Pose-Invariant 2.5D Face Recognition using Geodesic Texture Warping
}

\author{
Farshid Hajati $^{1,2}$, Abolghasem A. Raie ${ }^{1}$ \\ ${ }^{1}$ Faculty of Electrical Eng., Amirkabir University of \\ Technology, Tehran, Iran \\ ${ }^{2}$ School of Engineering, Griffith University, QLD 4111, \\ Australia \\ hajati@aut.ac.ir, raie@aut.ac.ir
}

\author{
Yongsheng $\mathrm{Gao}^{2,3}$ \\ ${ }^{2}$ School of Engineering, Griffith University, QLD 4111, \\ Australia \\ ${ }^{3}$ Queensland Research Lab, National ICT Australia, Australia \\ yongsheng.gao@griffith.edu.au
}

\begin{abstract}
In recent years, 3D face recognition has become a popular solution to deal with the problem of pose-invariant face recognition. The majority of 3D face data are, however, actually 2.5D which are sensitive to pose variations. This paper presents a novel Geodesic Texture Warping (GTW) solution for 2.5D poseinvariant face recognition. In this method, we use the geodesic distance computed on a $2.5 \mathrm{D}$ face scan to warp the texture of a rotated face to that of a frontal one to perform matching. A feasibility and effectiveness investigation for the proposed method is conducted using a wide range of experiments including samples with different face rotations. The encouraging experimental results demonstrate that the proposed method achieves much higher accuracy than the state-of-the-art method with a low computational cost.
\end{abstract}

Keywords-face recognition, pose, geodesic distance, 2.5D

\section{INTRODUCTION}

Face recognition has received significant attention in recent years due to its numerous potential applications in our societies such as personal identification, automatic surveillance, and human-computer interaction. Recently, using 3D information in face recognition systems has increased by the improvement in 3D scanners. The available 3D face scans used in face recognition can be divided into two categories. The first category consists of $2.5 \mathrm{D}$ images captured by commercial 3D scanners such as Minolta Vivid series [1]. These scanners capture 2.5D images from a single view of the face. A $2.5 \mathrm{D}$ image has the location of the face surface points in $3 \mathrm{D}$ space as at most one depth value (z direction) is available for every point in the $(x, y)$ plane [2]. In a $2.5 \mathrm{D}$ image some parts of the face may not be captured because of the self-occlusion. The second category consists of 3D models provided by merging several $2.5 \mathrm{D}$ images from different views and self-occlusion is trivial in this type of data. In real applications, 3D sensors can capture only partial views (2.5D images) of the objects. So, using the 2.5D images in face recognition researches is more acceptable.
While most efforts have been devoted to face recognition from 3D models, a few approaches have utilized 2.5D images. The proposed methods for 2.5D face recognition approaches can be divided into two categories: shape-based approaches and texture-based approaches. The first category consists of methods which just use the shape information extracted from the $2.5 \mathrm{D}$ images for recognition. In this category, using the shape information has been addressed in a number of different ways. Cartoux et al. [3] used facial profiles extracted from range images for face recognition. Gordon [4] suggested the feature extraction based on depth and curvature features. Tanaka et al. [5] considered the face recognition problem as a 3D shape recognition problem of rigid surfaces. Their method is based on the surface curvature information. Chua et al. [6] extended the use of point signature to recognize frontal face scans with expression changes. Hesher et al. [7] applied the PCA to the range images, and estimated the probability models for the coefficients. Pan et al. [8] utilized the partial directed Hausdorff distance to align and match two range images for verification.

The texture-based category consists of the algorithms which use the texture information to improve the recognition rate on 2.5D images. Change et al. [9] showed that the face recognition performance on texture and depth images is same. However, the combination of them can improve the recognition rate. Malassiotis and Strintzis [10] proposed a technique for face recognition using $2 \mathrm{D}$ and $3 \mathrm{D}$ data. They used 2.5D face images to compensate pose variations in corresponding texture images. They compensated the pose of input face images by matching the input $2.5 \mathrm{D}$ image with a reference model using the ICP algorithm [11]. The classification was performed using an Embedded Hidden Markov Model (EHMM) [12] on the compensated images. Lu et al. [2] used a matching technique between 2.5D face images and 3D face models for face recognition under pose variations. For each subject a reference 3D face model constructed by merging several $2.5 \mathrm{D}$ face images from different views. The recognition step consists of two components: a surface 
matching and an appearance matching. They used a modified ICP algorithm to match an input $2.5 \mathrm{D}$ image and the reference 3D models. Based on the surface matching's output, a candidates list from the gallery used for the appearance matching. Bronstein et al. [5, 6] proposed an expressioninvariant representation of faces called "canonical image". The canonical images are built by calculating the geodesic distances between points on the face surface. Mpiperis et al. [13] proposed a geodesic polar representation of the face surface. They used a geodesic-based isometric mapping to provide some warped texture images and apply PCA technique for classification. They reported that the performance of their algorithm is higher when it uses the depth information instead of the texture.

Different methods have been used to address the face recognition based on 2.5D images, but most of them have focused on only using the shape. In cases that they have used the texture, they used the texture as a small part in the face recognition system to improve the recognition rate on shape information. However, the texture plays an important role in the face recognition scenario, especially when the shapes of the faces in the gallery are similar.

In this paper, we present a novel pose-invariant $2.5 \mathrm{D}$ face recognition method using Geodesic Texture Warping (GTW). The GTW is robust to face rotations and highly efficient because of its low computational cost. The proposed method is evaluated by the publicly available FRAV3D database [14]. The performance of the proposed method is compared with the performance of the state-of-the-art method as the benchmark. Results demonstrate that the proposed method has much higher accuracy than the benchmark method.

The rest of this paper is organized as follows. The proposed Geodesic Texture Warping algorithm is described in section II. Section III presents the experimental results. Finally, conclusion is presented in Sections IV.

\section{Proposed Geodesic TeXture Warping}

The scheme of the proposed Geodesic Texture Warping (GTW) algorithm and the matching block is depicted in figure 1. There are three main steps in the GTW algorithm: 1) pointwise correspondence and geodesic computation, 2) texture warping, and 3) missing parts interpolation and cropping. In the following, we will describe the algorithm in detail.

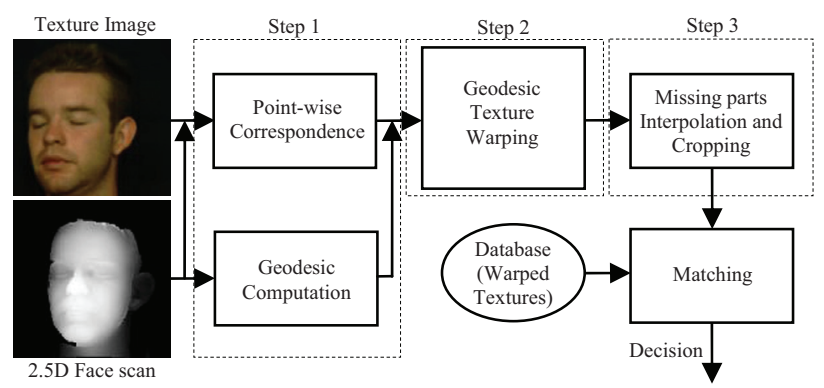

Figure 1. Proposed algorithm's scheme

\section{A. Point-wise Correspondence and Geodesic Computation}

First, the point-wise correspondence information between the input texture image and $2.5 \mathrm{D}$ face scan is computed. The raw 2.5D laser scans have some missing parts and spike noise especially in regions with hair. Therefore, we perform some surface smoothing and hole filling techniques to achieve a perfect $2.5 \mathrm{D}$ scan. Then, we interactively select three landmarks (nose tip and two eyes) on both input texture image and $2.5 \mathrm{D}$ scan. Since the angle views are same for $2.5 \mathrm{D}$ scan and texture image, these three landmarks are sufficient for the affine warping of the texture image to the $2.5 \mathrm{D}$ scan. Results obtained using this method may be considered close to the best results that one may obtain. Using this method, the correspondence information between the input texture and the input $2.5 \mathrm{D}$ scan is computed.

Second, the geodesic computation is performed on the face surface. Let $S$ be a surface, $P$ and $Q$ two points on $S$, as shown in figure 2. There are an infinite number of curves that belong to $S$ and connect $P$ with $Q$. The curve on the surface $S$ with the minimum length is called the "Geodesic Path" between two points and its length is called the "Geodesic Distance". In GTW, we just use the geodesic distance between face points.

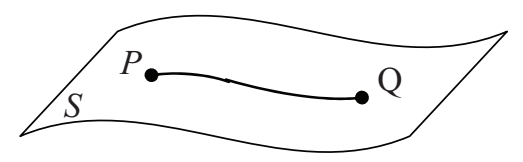

Figure 2. Geodesic path between P and Q

The computation of the geodesic distance on a surface involves the solving of Eikonal equation [15]

$$
|\nabla T|=1
$$

on the surface, where $T(Q)$ is the geodesic distance of the surface point $Q$ from a reference point (e.g. $P$ ). The main approach to solve the Eikonal equation is the Fast Marching Method (FMM) [16]. In a 2.5D space we can use the FMM in the triangular meshes, consisting of vertexes and faces, by the method proposed by Kimmel [17]. Consider a triangulation around a center grid point as shown in figure $3 \mathrm{a}$. The value of the center grid point is computed with the values of its neighbors in each triangle; it will be the smallest computed value from each triangle. The updating procedure for a triangle $A B C$ where $C$ is to be updated involves solving the following quadratic equation (see figure $3 b$ ).

$$
\begin{gathered}
\left(a^{2}+b^{2}-2 a b \cos \theta\right) t^{2}+2 b u(a \cos \theta-b) t+ \\
b^{2}\left(u^{2}-a^{2} \sin ^{2} \theta\right)=0
\end{gathered}
$$

where $u=T(B)-T(A)$ and $T(C)=T(A)+t$.

Using the above quadratic equation and FMM algorithm, we compute the geodesic distance for all vertexes in a triangular mesh from a reference point. In this case, we need 
to compute $N$ geodesic distances. Where, $N$ is the number of vertexes.

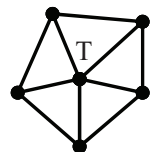

(a)

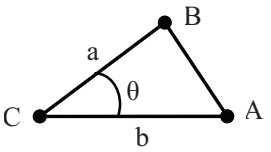

(b)
Figure 3. (a) A triangular mesh around a center grid point, (b) a triangle in the triangular mesh

In GTW, the nose tip is selected as the reference point and the geodesic distance of all face points are computed from the nose tip. To compute the geodesic distances, we need a $2.5 \mathrm{D}$ surface mesh. For this purpose, we apply a 2D Delaunay triangulation on $2.5 \mathrm{D}$ face scans to get a $2.5 \mathrm{D}$ mesh. After computing the geodesic distances for all face points, we normalize them by the geodesic distance of the two eyes from the reference point. The proposed normalization factor is $\gamma=\operatorname{avg}\left(D_{r}, D_{l}\right) / 70$ in which $D_{r}$ and $D_{l}$ are the geodesic distances of the right eye and the left eye from the nose tip, respectively.

\section{B. $\quad$ Texture Warping}

The second step in GTW is the warping of all face points from the texture of a rotated face to that of a frontal one based on the computations in the previous section. The warping function should be robust to the pose variations. The proposed warping function is $f:(x, y, z) \rightarrow(r, \varphi)$; where the $(x, y, z)$ represents the location of $2.5 \mathrm{D}$ scan points in the $2.5 \mathrm{D}$ space and $(r, \varphi)$ represents the location of warped texture point in a $2 \mathrm{D}$ polar coordinates. In the polar coordinates, $r$ and $\varphi$ are the radial coordinate and the angular coordinate, respectively. The function $f$ computes $r$ and $\varphi$ for each 2.5D scan point based on its location $(x, y$, and $z$ ). For the warping function $f$, the geodesic distance of each $2.5 \mathrm{D}$ scan point from the reference point (nose tip) is considered as its radial coordinate. The defined radial coordinate for each point remains constant under pose variations. To determine the angular coordinate for each $2.5 \mathrm{D}$ scan point, we need a reference plane. Using the reference plane, we can measure all angles in $2.5 \mathrm{D}$ space. In this paper, we define a reference plane using the location of the nose tip, right eye, and left eye in the $2.5 \mathrm{D}$ space (see figure 4a). First, we measure the Euclidean distance $d$ of the nose tip from the middle point between two eyes $(H)$. Then, we find two points with the distance $\hat{d}=k d$ from the two eyes in the direction $(\overrightarrow{N L} \times \overrightarrow{N H}) / \operatorname{norm}(\overrightarrow{N L} \times \overrightarrow{N H})$, where $k$ is a constant coefficient defined for the dataset by training. To train $k$, we measure $d$ and $\hat{d}$ for $n$ frontal face scans, manually. The objective criterion is that the result reference plane should be parallel to the $x-y$ plane in the $2.5 \mathrm{D}$ space. Then, we compute $k$ by

$$
k=\frac{1}{n} \sum_{i=1}^{n} \frac{\hat{d}_{i}}{d_{i}}
$$

The location of the reference plane is shown in figure $4 \mathrm{~b}$. For more visualization, we show the reference plane from the side view.

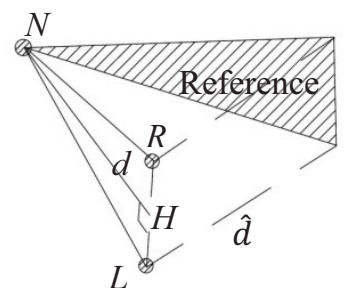

(a)

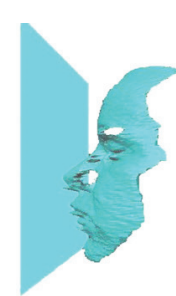

(b)
Figure 4. (a) Defining the reference plane using the nose tip $(N)$, right eye $(R)$, and the left eye $(L),(\mathrm{b})$ reference plane in $2.5 \mathrm{D}$ space

Using the reference plane, the angular coordinate of each face point is defined as the following: the angular coordinate of each point is the angle between two planes that are perpendicular to the reference plane. The first plane crosses the point and the nose tip and the second plane crosses the middle point between two eyes $(H)$ and the nose tip. All angles are measured counterclockwise from the second plane. Because of the fact that the location of face points is fixed respect to reference plane, the computed angular coordinate remains constant for each point in the presence of face rotations. Having the radial coordinate and angular coordinate of all face points and correspondence information between texture and 2.5D scan, we apply function $f$ to warp the texture of the rotated face to that of a frontal one. For this purpose, the location of each point in the warped texture image is found by its radial coordinate $(r)$ and angular coordinate $(\varphi)$ in the 2D polar coordinate. In this polar plane, the pole is the nose tip and the polar axis is drawn vertical and pointing upwards. All angles are measured counterclockwise from the polar axis.

\section{Missing Parts Interpolation and Cropping}

Because of self-occlusion, some parts of the warped texture image may be missed. Therefore, a symmetry-based interpolation method is used to reconstruct missing parts from the other side of the face. Finally, we crop the image to obtain a face rectangle which includes the most important facial features. For this purpose, a cropping model in that the distance of the nose tip is 80 pixels from the sides, 90 and 70 pixels from the top and the bottom, respectively, is used. Therefore, a $160 \times 160$ image is produced as the output.

\section{EXPERIMENTAL RESULTS}

In order to evaluate the performance of the GTW method in a pose-invariant face recognition system, an extensive experimental investigation is used covering face recognition under a wide range of different rotation angles. The experiments are conducted on the publicly available FRAV3D face database [14] consisting 106 subjects. Since our contribution is on the pose variations, we just use the frontal and rotated samples in our experiments. For this database, 
using a random set of 10 frontal 2.5D scans and (3) we set $k=$ 1.2. Some sample texture images from the dataset and their warped images by GTW algorithm are shown in figure 5.
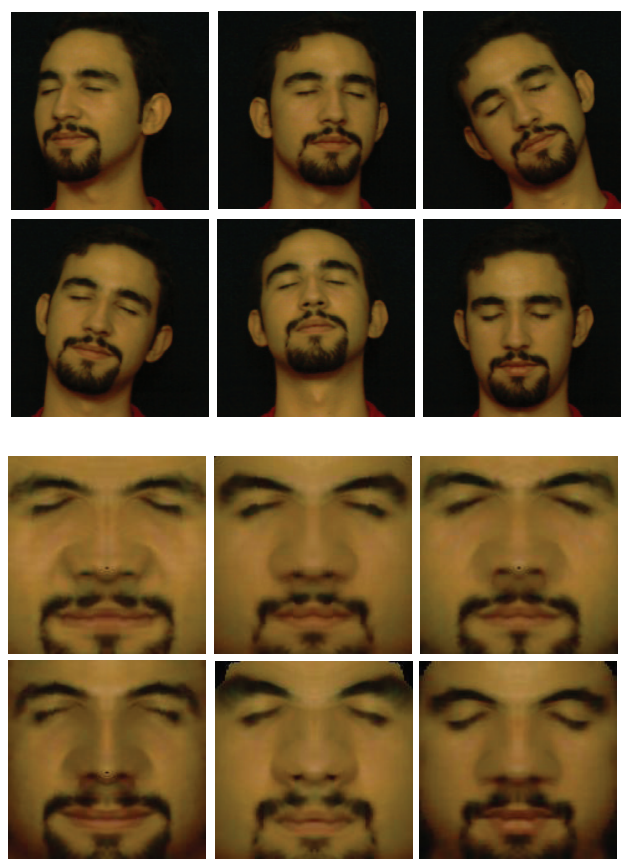

Figure 5. Some sample texture images and their warped images by the proposed algorithm

For each subject, a single frontal pair (texture image and $2.5 \mathrm{D}$ scan) is used as representative in the gallery set, while the rest images are used as probe images. In this paper, we use Principal Component Analysis (PCA) method [18] to classify warped texture images. We extract principal components and discard the first three most significant principal components which are responding sensibly to lighting variation. First, the images of training set (4 frontal views from each subject) are used to build an eigenspace. Then, each gallery and probe image is mapped to this eigenspace in the same way and the Euclidean distance between the probe and each gallery image is computed. The inverse of Euclidean distance is used as the matching criterion. To compare the performance of the proposed method, we also apply the method proposed by Malassiotis and Strintzis [10] as the benchmark on the same dataset. In each case, the matching results between each probe image and all gallery images are used to measure the rank-1 recognition rates and Cumulative Match Characteristics (CMC) curves. The rank-1 recognition rates for all rotations are listed in table I and the CMC curves are shown in figures 6-8.

As can be seen, the recognition rate of the GTW is higher than [10] for 5 out of 6 rotations (yaw-25 , yaw- $5^{\circ}$, rollsevere, roll-light, pitch-up). Especially for yaw $-25^{\circ}$ rotation, GTW performs much better (88.7 percent against to 44.3 percent). This demonstrates the capability of GTW method in handling large pose variation. Even in the worst case (pitch- down), our method is still comparable to [10] (88.7 percent against to 90.6). The overall average of recognition rates is 90.3 percent for the GTW against to the 79.1 percent for [10]. Therefore, the overall performance of GTW in handling pose is much higher than the [10]. Another advantage of the proposed method is its computational efficiency. We compute geodesic distances from just a single point (nose tip) to other face points. This allows us to use a denser $2.5 \mathrm{D}$ scan which is able to represent face shape more faithfully and reduce the warping error.

Table I. Rank-1 recognition rates (\%) for different rotations; yaw: rotation around $\mathrm{y}$-axis, roll: rotation around $\mathrm{z}$-axis, and pitch: rotation around $\mathrm{x}$-axis

\begin{tabular}{|c|c|c|c|c|c|c|c|}
\hline & $\begin{array}{c}\text { Yaw- } \\
25^{\circ}\end{array}$ & $\begin{array}{c}\text { Yaw- } \\
5^{\circ}\end{array}$ & $\begin{array}{c}\text { Roll- } \\
\text { Severe }\end{array}$ & $\begin{array}{c}\text { Roll- } \\
\text { Light }\end{array}$ & $\begin{array}{c}\text { Pitch- } \\
\text { Up }\end{array}$ & $\begin{array}{c}\text { Pitch- } \\
\text { Down }\end{array}$ & $\begin{array}{c}\text { Overall } \\
\text { (Avg.) }\end{array}$ \\
\hline $\begin{array}{c}\text { Proposed } \\
\text { Method }\end{array}$ & 88.7 & 92.5 & 90.6 & 92.5 & 88.7 & 88.7 & $\mathbf{9 0 . 3}$ \\
\hline $\begin{array}{c}\text { Malassiotis } \\
\text { method } \\
{[10]}\end{array}$ & 44.3 & 86.8 & 82.1 & 85.9 & 84.9 & 90.6 & $\mathbf{7 9 . 1}$ \\
\hline
\end{tabular}

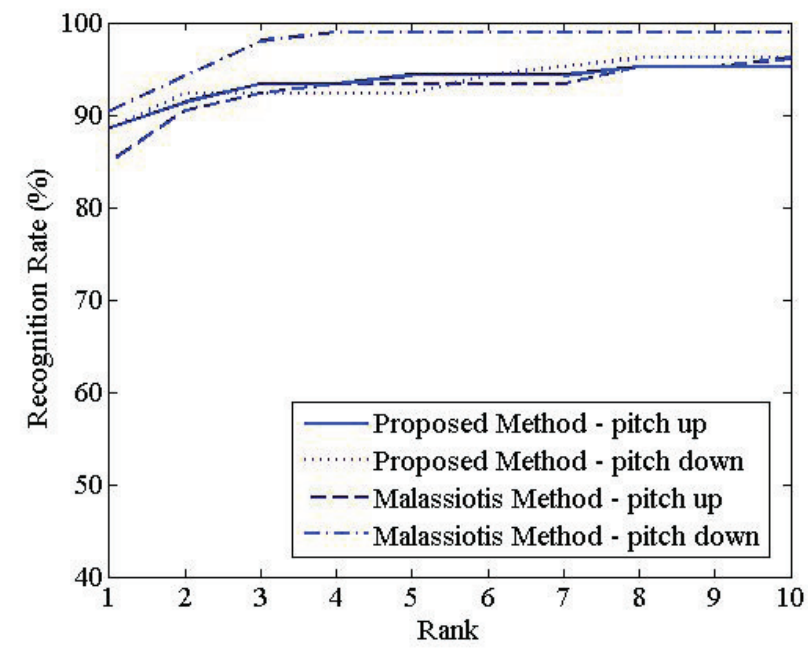

Figure 6. CMC curve for pitch rotations

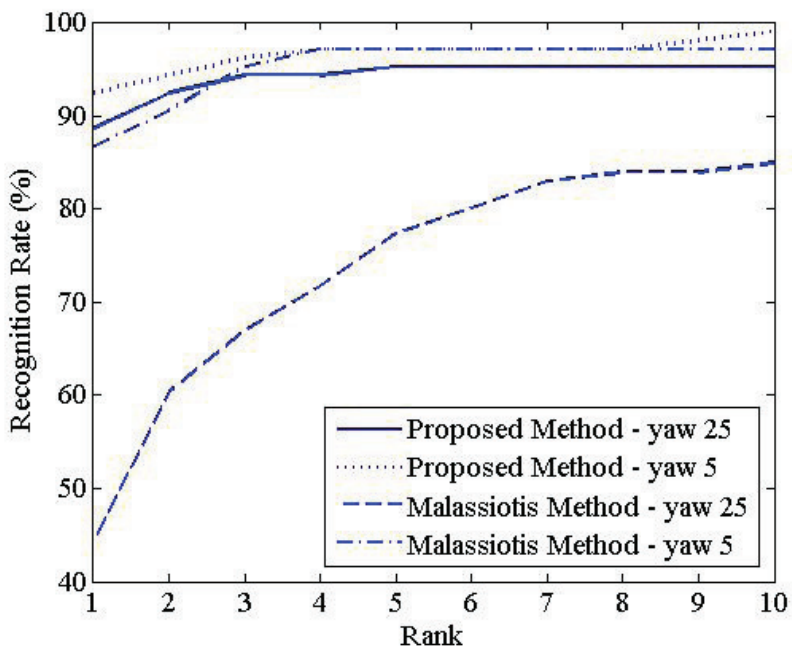

Figure 7. CMC curve for yaw rotations 


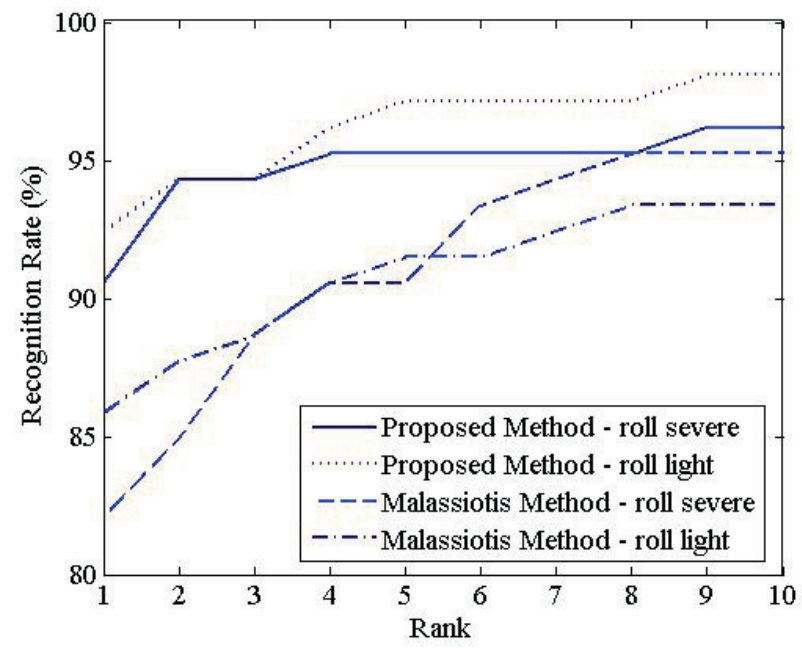

Figure 8. CMC curve for roll rotations

\section{CONCLUSION}

In this paper, we presented a novel texture warping method, named GTW, to recognize pose varying $2.5 \mathrm{D}$ faces. The proposed GTW method uses the geodesic information to warp the texture on a rotated $2.5 \mathrm{D}$ face image to that of a frontal one to perform matching. The GTW compared to the state-of-theart method as the benchmark on the publically available FRAV3D database. The performance of the GTW is much higher than the benchmark method. The overall rank-1 recognition rate is 90.3 percent for GTW, while it is 79.1 percent for the benchmark method. Furthermore, the GTW is an efficient method from the computational cost view.

\section{REFERENCES}

[1] Minolta vivid, http://www.konicaminolta.com/sensingusa/products/3d.

[2] X. Lu, A. K. Jain, and D. Colbry, "Matching $2.5 \mathrm{~d}$ face scans to $3 \mathrm{~d}$ models," IEEE Trans. on Pattern Analysis and Machine Intelligence, vol. 28, no. 1, pp. 31-43, 2006.

[3] J. Y. Cartoux, J. T. LaPreste, and M. Richetin, "Face authentication or recognition by profile extraction from range images," Proc. of the Workshop Interpretation of 3D Scenes, pp. 194-199, 1989.

[4] G. G. Gordon, "Face recognition based on depth and curvature features," Proc. of the IEEE Conference on Computer Vision and Pattern Recognition, pp. 108-110, 1992.

[5] H. T. Tanaka, M. Ikeda, and H. Chiaki, "Curvature-based face surface recognition using spherical correlation-principal directions for curved object recognition," Proc. of the 3rd. International Conference on Face and Gesture Recognition, pp. 372-377, 1998.

[6] C. Chua, F. Han, and Y. Ho, "3d human face recognition using point signature," Proc. of the 4th International Conference on Automatic Face and Gesture Recognition, pp. 233-238, 2000.

[7] C. Hesher, A. Srivastava, and G. Erlebacher, "Principal component analysis of range images for facial recognition," Proc. of the International Multiconference on Computer Science and Information Systems, pp. 62-68, 2002.

[8] G. Pan, Z. Wu, and Y. Pan, "Automatic 3d face verification from range data," Proc. of the IEEE International Conference on Acoustics, Speech, and Signal Processing, pp. 193-196, 2003.

[9] K. Chang, K. Bowyer, and P. Flynn, "Multi-modal 2d and 3d biometrics for face recognition," Proc. of the IEEE International Workshop on Analysis and Modeling of Faces and Gestures, pp. 187-195, 2003.
[10] S. Malassiotis, and M. G. Strintzis, "Robust face recognition using 2d and 3d data: Pose and illumination compensation," Pattern Recognition, vol. 38, no. 12, pp. 2537-2548, 2005.

[11] P. Besl, and N. McKay, "A method for registration of $3 \mathrm{~d}$ shapes," IEEE Trans. on Pattern Analysis and Machine Intelligence, vol. 14, no. 2, pp. 239-256, 1992.

[12] L. R. Rabiner, "Tutorial on hidden markov models and selected applications in speech recognition," Proc. IEEE, vol. 77, no. 2, pp. 267296, 1989.

[13] I. Mpiperis, S. Malassiotis, and M. G. Strintzis, "3-d face recognition with the geodesic polar representation," IEEE Trans. on Information Forensics and Security, vol. 2, no. 3, pp. 537-547, 2007.

[14] Frav3d, http://www.frav.es/databases/FRAV3D/.

[15] Y. R. Tsai, L. Cheng, S. Osher, and H. Zhao, "Fast sweeping algorithms for a class of hamilton-jacobi equations," SIAM Journal on Numerical Analysis, vol. 41, no. 2, pp. 673-694, 2003.

[16] J. A. Sethian, Level set methods and fast marching methods evolving interfaces in computational geometry, Fluid mechanics, computer vision, and materials science, Cambridge: Cambridge University Press, 1999.

[17] R. Kimmel, and J. A. Sethian, "Computing geodesic paths on manifolds," Applied Mathematics, vol. 95, no. 15, pp. 8431-8435, 1998.

[18] M. Turk, and A. Pentland, "Eigenfaces for recognition," Journal of Cognitive Neuroscience, vol. 3, no. 1, pp. 71-86, 1991. 